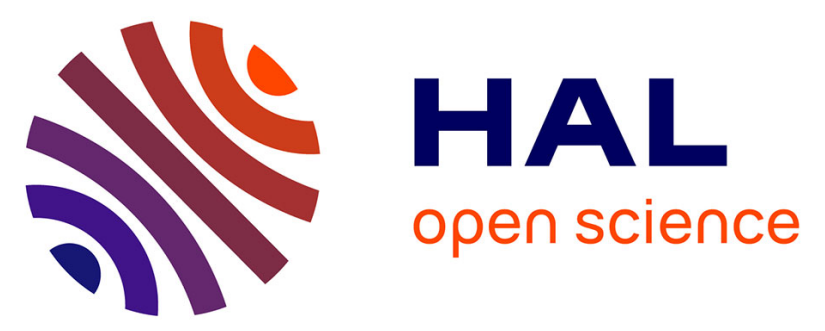

\title{
Extension of modal reduction methods to non-linear coupled structure-acoustic problems
}

Youssef Gerges, Emeline Sadoulet-Reboul, Morvan Ouisse, Nourredine

Bouhaddi

\section{- To cite this version:}

Youssef Gerges, Emeline Sadoulet-Reboul, Morvan Ouisse, Nourredine Bouhaddi. Extension of modal reduction methods to non-linear coupled structure-acoustic problems. Revue Européenne de Mécanique Numérique/European Journal of Computational Mechanics, 2011, 20 (1-4), pp.227 - 245. 10.3166/ejcm.20.227-245 . hal-01511278

\section{HAL Id: hal-01511278 \\ https://hal.science/hal-01511278}

Submitted on 20 Apr 2017

HAL is a multi-disciplinary open access archive for the deposit and dissemination of scientific research documents, whether they are published or not. The documents may come from teaching and research institutions in France or abroad, or from public or private research centers.
L'archive ouverte pluridisciplinaire HAL, est destinée au dépôt et à la diffusion de documents scientifiques de niveau recherche, publiés ou non, émanant des établissements d'enseignement et de recherche français ou étrangers, des laboratoires publics ou privés.

\section{(c)(1)}

Distributed under a Creative Commons Attribution| 4.0 International License 


\title{
Extension of modal reduction methods to non-linear coupled structure-acoustic problems
}

\author{
Youssef Gerges - Emeline Sadoulet-Reboul \\ Morvan Ouisse - Noureddine Bouhaddi
}

\author{
Institut FEMTO-ST, UMR 6174, Département de Mécanique Appliquée \\ Université de Franche-Comté \\ 24 rue de l'Epitaphe, \\ F-25000 Besançon \\ youssef.gerges@edu.univ-fcomte.fr
}

ABSTRACT. This paper proposes a robust reduction method dedicated to non-linear vibroacoustic problems in the context of localized geometrical non-linearities. The method consists in enriching the truncated uncoupled modal basis of the linear model by a static response due to unit forces on the non-linear degrees of freedom and by the static response of the fluid due to the interaction with the structure. To show the effectiveness of the proposed method, numerical simulations of responses of an elastic plate closing an acoustic cavity and a hang-on exhaust are performed.

RÉSUMÉ. Cet article propose une méthode de réduction robuste dédiée aux problèmes vibroacoustiques non linéaires. Le type de non-linéarité envisagé est géométrique localisé. La méthode de réduction introduite consiste à enrichir la base modale tronquée du système découplé sans non-linéarité par des réponses statiques de la structure dues à des efforts unitaires sur les degrés de liberté non linéaires ainsi que par la réponse statique du fluide induite par le couplage avec la structure. Les réponses temporelles d'une plaque appuyée sur une cavité acoustique et d'un tuyau sonore suspendu sont simulées pour montrer l'efficacité de la méthode proposée.

KEYWORDS: reduced model, fluid structure interaction, non-linear vibration, vibroacoustic coupling, time integration.

MOTS-CLÉS : réduction de modèle, interaction fluide structure, vibration non linéaire, couplage vibroacoustique, intégration temporelle. 


\section{Introduction}

This paper presents a kind of method for model reduction dedicated to non-linear internal vibroacoustic problems. Modeling this problem still remains a heavy exercise for industrial applications. The starting point is the formulation choice; structural displacement and acoustic pressure $(u, p)$ are employed in this paper. Using the finite element method (FEM) leads to an unsymmetrical matrix system with large dimensions. Symmetric formulation can also be obtained using mathematical techniques leading to full matrices (Irons, 1970), or alternative formulation choices (Morand et al., 1992; Tran, 2009) which are not relevant for damping introduction.

The unsymmetric character of the system associated to its large size implies high computational times which are not compatible with optimization and robustness analysis. In this context, model reduction using projection bases is one of the ways to reduce the calculation cost. Most of the applications in literature do not consider the non-linear behaviour which is found in many examples. The few of them treating nonlinear effects use specific methods dedicated to non-linear dynamics. In particular the proper orthogonal decomposition or the non-linear normal modes can be efficiently applied to obtain a reduced model. (Amabili et al., 2007; Amabili, 2008). The use of the harmonic balance method in a frequency study is found in many applications (Nayfeh et al., 1995). Transient analysis require the implementation of an explicit or implicit integration scheme (Géradin et al., 1997; Bathe, 1982). For external vibroacoustics, an iterative procedure dedicated to FEM/BEM coupling including non-linear effects on the structure has been proposed by (Soares-Jr et al., 2005).

This study is based on the development of a reduction basis dedicated to internal non-linear vibroacoustic problems where geometrical non-linearities are localized. The formulation of the non-linear vibroacoustic problem with structural displacements and acoustic pressure formulation is first presented. Reduced order model of the considered problem is presented in section 3 including an original association of the uncoupled Ritz basis with both coupling and non-linear effects. Newmark algorithm dedicated to time integration is studied in section 4 (Géradin et al., 1997; Bathe, 1982). Section 5 presents the predictor indicators used for comparison with the full model. Section 6 is finally dedicated to numerical illustrations of the proposed methodology.

\section{Formulation of non-linear vibroacoustic problem}

This section presents the formulation of the non-linear vibroacoustic problem. The starting point is the variational formulation leading to the finite element formulation of the problem.

The vibroacoustic problem considered in this work is presented in Figure 1. Let us consider a fluid-filled domain $\Omega_{f}$ coupled with a structure $\Omega_{s}$ presenting large displacements. $\Gamma_{f s}$ is the coupling surface. The structure is submitted to volume and surface loads $f_{v}(t)$ and $f_{s}(t)$. 


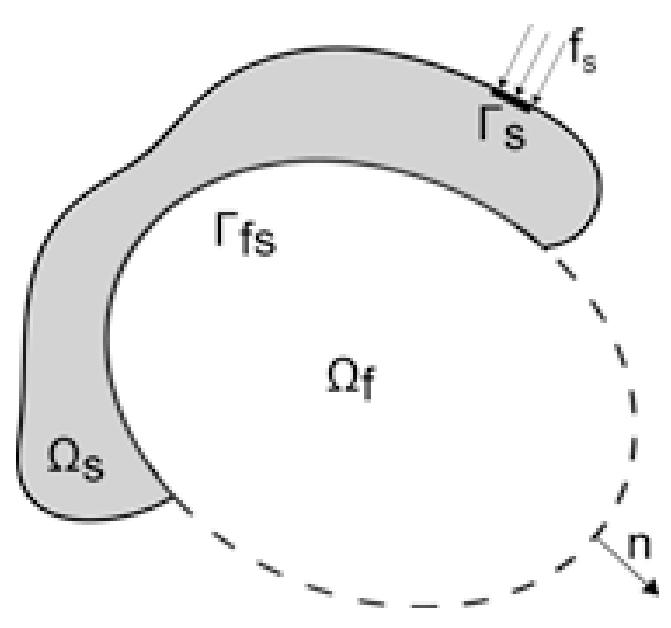

Figure 1. Vibroacoustic problem

\subsection{Structural-acoustic formulation}

The structural equation of motion is deduced from the virtual work principle written as:

$$
\delta \int_{t_{1}}^{t_{2}}(U+T) d t=0
$$

where:

$-T$ is the kinetic energy:

$$
T=\frac{1}{2} \int_{\Omega_{s}} \rho_{s} \dot{u} \dot{u} d \Omega
$$

$\rho_{s}$ being the structural density and $u$ being the structural displacement.

$-U$ is the potential energy defined as the sum of the strain energy $U_{\text {strain }}$ and the potential energy due to the applied load $U_{\text {pot }}$ :

$$
\begin{aligned}
& U_{\text {pot }}=-\int_{\Omega_{s}} f_{v}(t) u d \Omega-\int_{\Gamma_{s}} f_{s}(t) u d \Gamma, \\
& U_{\text {strain }}=\frac{1}{2} \int_{\Omega_{s}} \tau^{T} D \tau d \Omega .
\end{aligned}
$$

$D$ is the material stiffness matrix and $\tau$ is the $2^{n d}$ order Green Lagrange strain tensor related to the displacement field. Large displacement theory with small strain is considered. This leads to a strain tensor written as follow:

$$
\tau(u)=\underbrace{\frac{1}{2}\left(\nabla u+\nabla u^{t}\right)}_{\tau^{l}}+\underbrace{\frac{1}{2} \nabla u^{t} \nabla u}_{\tau^{n l}},
$$

where $\tau^{l}$ and $\tau^{n l}$ respectively represent the linear and non-linear parts of the tensor. 
The constitutive equation of the linear-elastic material is:

$$
S=D \tau,
$$

where $S$ is the $2^{\text {nd }}$ Piola-Kirchhoff stress tensor.

Writing the virtual work principle leads to the weak formulation of the problem associated to the structure (Morand et al., 1992; Pérignon, 2004):

$$
\begin{array}{r}
\int_{\Omega_{s}} S^{T} \tau^{l}(\delta u) d \Omega+\int_{\Omega_{s}} S^{T} \tau^{n l}(u, \delta u) d \Omega+\int_{\Omega_{s}} \rho_{s} \frac{\partial^{2} u}{\partial t^{2}} \delta u d \Omega= \\
\int_{\Omega_{s}} f_{v} \delta u d \Omega+\int_{\Gamma_{s}} f_{s} \delta u d \Gamma+\int_{\Gamma_{f s}} p n \delta u d \Gamma .
\end{array}
$$

The term $\int_{\Gamma_{f s}} p n \delta u d \Gamma$ corresponds to the action of the fluid on the structure, $\mathrm{p}$ being the acoustic pressure.

Concerning the fluid domain $\Omega_{f}$, the equilibrium state is expressed by the equation:

$$
\Delta p=\frac{1}{c^{2}} \frac{\partial^{2} p}{\partial t^{2}}
$$

where $c$ is the sound of speed in the fluid.

The boundary condition applied on the coupling area $\Gamma_{f s}$ corresponds to the sliding condition:

$$
-\frac{\partial p}{\partial n}=\rho_{f} \frac{\partial^{2} u}{\partial t^{2}}
$$

where $\rho_{f}$ is the density of the fluid.

By applying the Green formula, the variational formulation of the fluid can be written as (Morand et al., 1992):

$$
\frac{1}{\rho_{f}} \int_{\Omega_{f}} \nabla p \nabla \delta p d \Omega+\frac{1}{\rho_{f} c^{2}} \int_{\Omega_{f}} \frac{\partial^{2} p}{\partial t^{2}} \delta p d \Omega+\int_{\Gamma_{f s}} \frac{\partial^{2} u}{\partial t^{2}} n \delta p d \Gamma=0 .
$$

Coupling Equations [7] and [10] leads to:

$$
\left\{\begin{array}{r}
\int_{\Omega_{s}} S^{T} \tau^{l}(\delta u) d \Omega+\int_{\Omega_{s}} S^{T} \tau^{n l}(u, \delta u) d \Omega+\int_{\Omega_{s}} \rho_{s} \frac{\partial^{2} u}{\partial t^{2}} \delta u d \Omega- \\
\int_{\Gamma_{f s}} p n \delta u d \Gamma=\int_{\Gamma_{s}} f_{s} \delta u d \Gamma+\int_{\Omega_{s}} f_{v} \delta u d \Omega \\
\int_{\Omega_{f}} \nabla p \nabla \delta p d \Omega+\frac{1}{c^{2}} \int_{\Omega_{f}} \frac{\partial^{2} p}{\partial t^{2}} \delta p d \Omega+\rho_{f} \int_{\Gamma_{f s}} \frac{\partial^{2} u}{\partial t^{2}} n \delta p d \Gamma=0 .
\end{array}\right.
$$




\subsection{Finite element formulation}

Using the finite element method, the problem can be written as follows:

$$
\left[\begin{array}{cc}
M_{s} & 0 \\
\rho_{f} C^{T} & M_{f}
\end{array}\right]\left[\begin{array}{l}
\ddot{U} \\
\ddot{P}
\end{array}\right]+\left[\begin{array}{cc}
K_{s}(U) & -C \\
0 & K_{f}
\end{array}\right]\left[\begin{array}{l}
U \\
P
\end{array}\right]=\left[\begin{array}{c}
F \\
0
\end{array}\right],
$$

where:

$$
\left\{\begin{array}{l}
M_{s} \rightarrow \int_{\Omega_{s}} \rho_{s} \frac{\partial^{2} u}{\partial t^{2}} \delta u d \Omega, \\
K_{s}(U) \rightarrow \int_{\Omega_{s}} S^{T} \tau^{l}(\delta u) d \Omega+\int_{\Omega_{s}} S^{T} \tau^{n l}(u, \delta u) d \Omega \\
M_{f} \rightarrow \frac{1}{c^{2}} \int_{\Omega_{f}} \frac{\partial^{2} p}{\partial t^{2}} \delta p d \Omega \\
K_{f} \rightarrow \int_{\Omega_{f}} \nabla p \nabla \delta p d \Omega \\
C \rightarrow \int_{\Gamma_{f s}} p n \delta u d \Gamma \\
F \rightarrow \int_{\Gamma_{s}} f_{s} \delta u d \Gamma
\end{array}\right.
$$

$-M_{s}$ is the mass matrix of the structure.

$-K_{s}$ is the stiffness matrix of the structure. $K_{s}$ is a function of $U$; for geometrical non-linearities it is the sum of a linear term arising from the linear problem $K_{s}^{l}$ and a non-linear term. For localized non-linearity case, $K_{s}$ can be written as:

$$
K_{s}(U)=K_{s}^{l}+K_{s}^{n l} \operatorname{diag}(U)^{n-1},
$$

where $K_{s}^{n l}$ is the hardening coefficient of the non-linearity and $n$ is the degree of non-linearity.

$-M_{f}$ and $K_{f}$ are the matrix corresponding respectively to the discretization of kinematic energy and the compressibility of the fluid.

$-C$ is the coupling matrix corresponding to the action of the structure on the fluid or vice versa.

$-F$ is the structure excitation function of the time $t$.

The matrix system can be expressed as a differential equation written as:

$$
M \ddot{X}+K(X) X=f(t) .
$$

Solving this kind of equation depends on the nature of $f(t)$. Using the modal decomposition method to diagonalize the problem is not possible, this is due to the non-linear character of the system.

If $f(t)$ is harmonic, one of the efficient techniques to solve the problem is the harmonic balance method (Nayfeh et al., 1995).

Using an arbitrary excitation requires the implementation of time integration method (Géradin et al., 1997; Bathe, 1982).

In both cases, solving the problem is time consuming. In the next section, a reduced order method dedicated to this kind of problem is presented. The projecting basis should take into account both non-linear and coupling effects. 


\section{Reduced order method}

This section presents the strategy used for elaborating the reduced base. The starting point is the classical approach based on the creation of the reduced Ritz basis issued from the uncoupled problems. Non-linear and coupling effects are considered as a perturbation of the non-coupled problem. Enriching the basis by residues issued from these perturbations leads to a robust reduced basis dedicated to vibroacoustic problems. The original contribution of this work is in the combination of different bases associated to a singular value decomposition to ensure good conditioning.

\subsection{Uncoupled modal basis}

As it was mentioned in the previous paragraph, a reduced order method is required for modeling non-linear vibroacoustic problem. The proposed basis should be robust and easy to implement. A first approximation model corresponds to the use of an uncoupled modal basis issued from the in vacuo linear structural problem $\left(K_{s}^{n l}=0\right)$ and the rigid wall cavity problem. This leads to a finite element approximation written as follows:

$$
\left[\begin{array}{l}
U \\
P
\end{array}\right] \approx\left[\begin{array}{cc}
T_{s m b} & 0 \\
0 & T_{f m b}
\end{array}\right]\left[\begin{array}{l}
q_{s} \\
q_{f}
\end{array}\right],
$$

where $T_{s m b}$ and $T_{f m b}$ respectively represent the truncated structure and fluid modal bases.

\subsection{Non-linear enriching}

Localized non-linear behaviour is considered as a perturbation modifying the linear response. This perturbation is assimilated to a residual excitation force. In order to take into account this force in the non-linear reduced model, a linear static response of the in vacuo structure due to a unit load on each non-linear degree of freedom is considered:

$$
\Delta T_{s n l}^{i}=\left(K_{s}^{l}\right)^{-1} f^{i},
$$

where $i$ is the $i^{\text {th }}$ non-linear degree of freedom and

$$
f^{i}=[0 \ldots 1 \ldots 0]^{T} .
$$

Orthogonalization is necessary to ensure good conditioning of the problem. This is realized with a singular value decomposition $(S V D)$ :

$$
T_{s}=\left[T_{s m b} \mid \Delta T_{s n l}\right]_{S V D} .
$$

This leads to a new finite element approximation written as follows:

$$
\left[\begin{array}{l}
U \\
P
\end{array}\right] \approx\left[\begin{array}{cc}
T_{s} & 0 \\
0 & T_{f m b}
\end{array}\right]\left[\begin{array}{l}
q_{s} \\
q_{f}
\end{array}\right] .
$$




\subsection{Coupling enriching}

The main goal of this section is to propose a modal synthesis method that can take into account coupling effects. The homogeneous matrix formulation associated to the Equation [12] in the frequency domain is written as:

$$
\left(\left[\begin{array}{cc}
K_{s}(U) & -C \\
0 & K_{f}
\end{array}\right]-\omega^{2}\left[\begin{array}{cc}
M_{s} & 0 \\
\rho_{f} C^{T} & M_{f}
\end{array}\right]\right)\left[\begin{array}{l}
U \\
P
\end{array}\right]=\left[\begin{array}{l}
0 \\
0
\end{array}\right] .
$$

The term $\rho_{f} C^{T} U$ can be interpreted as an excitation of the fluid due to the structure. For the fluid part, we have :

$$
\left(K_{f}-\omega^{2} M_{f}\right) P=\omega^{2} \rho_{f} C^{T} U .
$$

This excitation is not known but it can be approximated by projecting the displacement on the structural basis introduced in the previous section [19]. Updating the fluid basis by including this response leads to a new reduced basis. This new residual basis is written as (Tran, 2009; Tran et al., 2010):

$$
\Delta T_{f s}=\left(K_{f}-\omega_{c}^{2} M_{f}\right)^{-1} C^{T} T_{s},
$$

and should be decomposed in singular value to ensure orthogonality:

$$
\left[\begin{array}{l}
U \\
P
\end{array}\right] \approx\left[\begin{array}{cc}
T_{s} & 0 \\
0 & T_{f}
\end{array}\right]\left[\begin{array}{l}
q_{s} \\
q_{f}
\end{array}\right]
$$

where:

$$
T_{f}=\left[T_{f m b} \mid \Delta T_{f s}\right]_{S V D} .
$$

\subsection{Coupling with heavy fluid}

The reduced order method presented above is dedicated to the non-linear vibroacoustic problem with light coupling. In the case where the fluid density can not be neglected compared to the structure, the coupling is considered strong. The fluid behaviour impacts the structure and should be considered in the modal synthesis. Linear static response of the structure due to the fluid effect is written as:

$$
U=\left(K_{s}^{l}\right)^{-1} C P .
$$

This excitation is not known but it is approximated by projecting the pressure $P$ on the fluid basis introduced in the section bellow [25]. Enriching the structural basis by the static residual response due to the heavy fluid impact leads to a new basis written as follows (Tran, 2009; Tran et al., 2010):

$$
\left[\begin{array}{l}
U \\
P
\end{array}\right] \approx\left[\begin{array}{cc}
T_{s h f} & 0 \\
0 & T_{f}
\end{array}\right]\left[\begin{array}{l}
q_{s} \\
q_{f}
\end{array}\right]
$$


where

$$
T_{s h f}=\left[T_{s} \mid \Delta T_{s f}\right]_{S V D}
$$

and

$$
\Delta T_{s f}=K_{s}^{l-1} C T_{f}
$$

Once the reduced basis has been established, the model reduction is performed as follows:

$$
X=\left[\begin{array}{l}
U \\
P
\end{array}\right]=T\left[\begin{array}{l}
q_{s} \\
q_{f}
\end{array}\right]=T q
$$

$T$ is the reduction basis defined in the sections above. Dynamics equilibrium defined in Equation [15] becomes:

$$
M_{r} \ddot{q}+K_{r}(q) q=f_{r}(t),
$$

where:

- $M_{r}$ is the reduced mass matrix: $M_{r}=T^{T} M T$

$-K_{r}$ is the reduced stiffness matrix: $K_{r}=T^{T} K T$

$-f_{r}$ is the reduced force vector: $f_{r}=T^{T} f$

\section{Computation of non-linear temporal response: Newmark algorithm}

In this section, the well known Newmark algorithm is recalled. As it was mentioned before, the strategy to solve the dynamic equations depends on the excitation. Equilibrium equation in presence of dissipative energy modeled by a damping matrix $D$ is written as:

$$
r(X)=M \ddot{X}(t)+D \dot{X}(t)+K(X) X(t)-f(t)=0 .
$$

In the case of an arbitrary excitation, numerical integration in the time domain is required. It consists in calculating the iterative state of the system (displacement, velocity and acceleration) as a function of time progress. A widely used technique is the Newmark algorithm; compared to other numerical integration, the Newmark algorithm is relatively stable. It uses the following state description:

$$
A_{n}=\left[\begin{array}{c}
X_{n} \\
\dot{X}_{n} \\
\ddot{X}_{n}
\end{array}\right]=\left[\begin{array}{c}
X\left(t_{n}\right) \\
\dot{X}\left(t_{n}\right) \\
\ddot{X}\left(t_{n}\right)
\end{array}\right],
$$

where $A_{n}$ represents the system state calculated at time $t_{n}$. $A_{n+1}$ represents the system state at one time step later, it is written as (Géradin et al., 1997; Bathe, 1982):

$$
\left\{\begin{array}{l}
X_{n+1}=X_{n}+h \dot{X}_{n}+h^{2}\left(\frac{1}{2}-\beta\right) \ddot{X}_{n}+h^{2} \beta \ddot{X}_{n+1} \\
\dot{X}_{n+1}=\dot{X}_{n}+(1-\gamma) h \ddot{X}_{n}+\gamma h \ddot{X}_{n+1} \\
\ddot{X}_{n+1}
\end{array}\right.
$$


where $h$ is the chosen time step, $\gamma$ and $\beta$ are the parameters of the Newmark algorithm. $\ddot{X}_{n+1}$ is calculated through the dynamic Equation [32].

Non-linear behaviour can lead to bad predictions. A residue evaluation is considered to ensure dynamic equilibrium. The residual equation is evaluated at each time step through the linearized first order equation:

$$
r_{n+1}+S_{n+1}^{i} \Delta X=0,
$$

where $S_{n+1}^{i}=\left[\frac{\partial r}{\partial X}\right]_{X_{n+1}^{i}}=K^{t}+\frac{\gamma}{\beta h} D^{t}+\frac{1}{\beta h^{2}} M$ is the Jacobian matrix of $r ; K^{t}$ and $D^{t}$ are respectively the Jacobian matrices of $K$ and $D$ while $\Delta X=-\left(S_{n+1}^{i}\right)^{-1} r_{n+1}$ is the displacement correction. $i$ is the $i^{\text {th }}$ iteration of the residue evaluation (correction). Experience shows that for a null acceleration at the beginning of every iteration, and by correcting the approximation during iterations, this can provide stable and fast process. For the same reason, a constant Jacobian matrix can be used in the correction algorithm. The advantage compared to the evaluation of the Jacobian matrix at each step is the inversion procedure. Otherwise, convergence to the equilibrium state will be longer. Choosing one of these techniques will depend on the application.

The same algorithm is used for the integration of the reduced model [31]. Matrices $M, K$ and $D$ and vectors $X$ and $f$ are replaced by their corresponding reduction $\left(M_{r}\right.$, $K_{r}, D_{r}, q$ and $f_{r}$ respectively). Once a reduced state is evaluated, physical response is performed using Equation [30].

The numerical integration algorithm is resumed as follows:

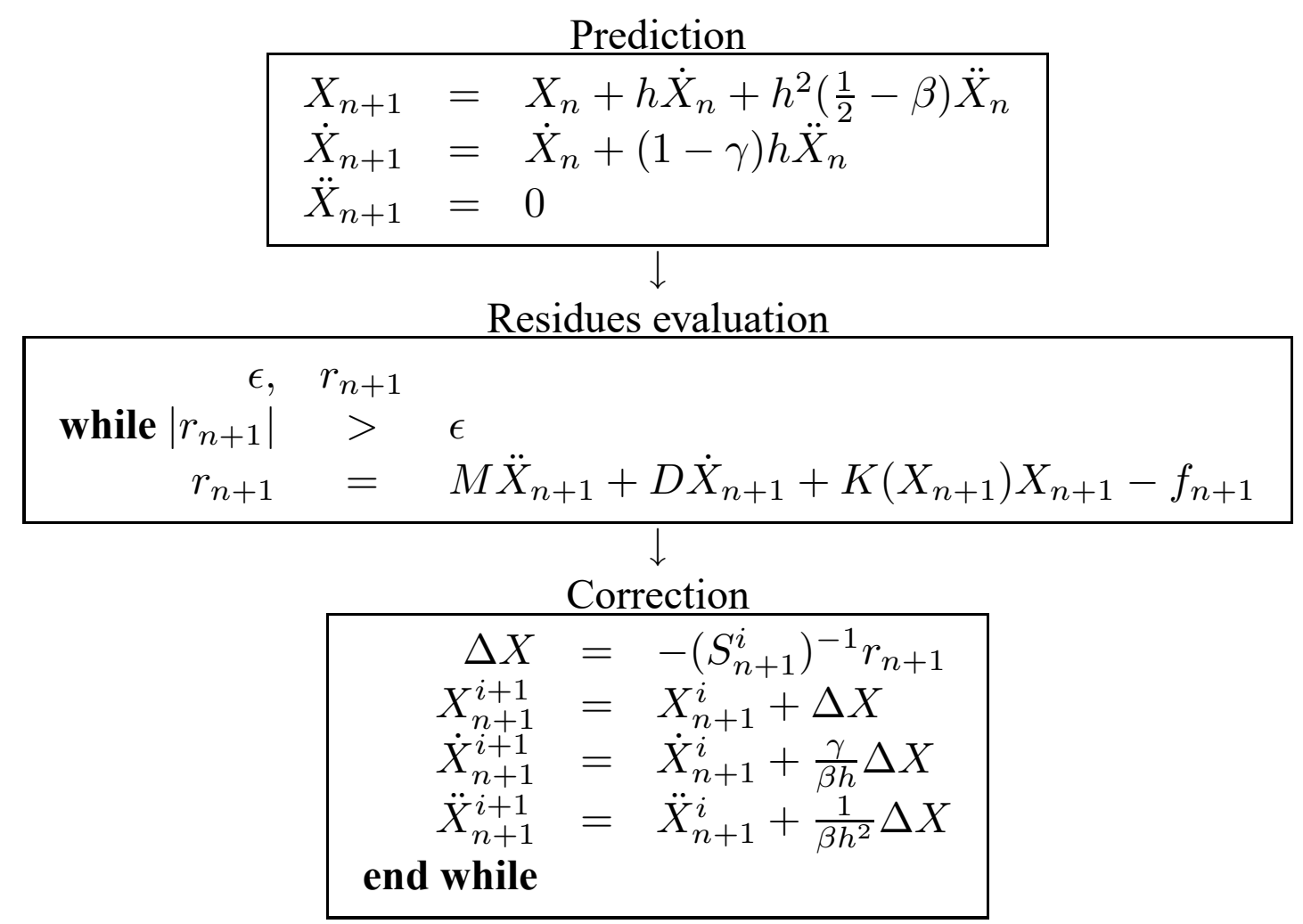




\section{Prediction indicators}

Results comparison tools are based on statistic indicators associated to the structure and fluid responses in addition to the energy indicators that result from acoustic and kinetic energies.

\subsection{Temporal moments}

Temporal moments are used in transient responses in order to quantify the comparison between different models. It is used to qualify the model response. The $i^{t h}$ order of the temporal moment of a response $y(t)$ is defined as (Masson et al., 2006):

$$
M_{i}=\int_{-\infty}^{+\infty}\left(t-t_{s}\right)^{i}(y(t))^{2} d t
$$

where $t_{s}$ represents the temporal shift and $i$ the moment index order.

In this work, the following temporal moment $M_{i}$ is used with $t_{s}=0$ and normalized as follows:

$$
\left\{\begin{array}{l}
E=M_{0}: \text { Energy }\left(m^{2} s\right) \\
T=\frac{M_{1}}{M_{0}}: \text { Central time (centroid) }(s), \\
D^{2}=\frac{M_{2}}{M_{0}}-\left(\frac{M_{1}}{M_{0}}\right)^{2}: \text { Root mean square duration }\left(s^{2}\right) .
\end{array}\right.
$$

\subsection{Energy indicators}

The indicators that are used are the acoustic energy and the mean square velocity. The acoustic energy is defined as the sum of the kinetic and potential energies in the fluid domain. The discretized form is expressed as follows:

$$
E_{a}=\frac{1}{2 \rho_{f}} P K_{f} P+\frac{1}{2 \rho_{f}} \dot{P} M_{f} \dot{P} .
$$

The discretized form of the mean square normal velocity is defined as:

$$
{\overline{V_{n}}}^{2}=\frac{1}{\left|S_{s}\right|} V M_{v_{n}} V,
$$

where $M_{v_{n}}$ comes from the discretization of $\int v_{n}^{2} d S$.

\section{Numerical illustration}

In order to illustrate the proposed idea, two applications are presented in this section. A parallelepipedic acoustic cavity fixed with a thin plate presenting non-linear 
localized behaviour and an exhaust filled of air with non-linear links. Two types of excitation are studied. For the acoustic cavity, the response of the system due to an impact of $500 \mathrm{~N}$ is considered. For the exhaust, a sweep sine in the frequency band of the model is exciting the system. The full model response is compared to the results obtained with several reduction bases. The reduced order methods used in these applications are the following:

- reduced model using the uncoupled modal basis of the system (Modal basis);

- enrichment of the uncoupled structural modal basis by the static response of the structure due to the unit forces on the non-linear degrees of freedom (NL residues);

- enrichment of the uncoupled fluid basis only by the static response of the fluid due to the presence of the structure (Coupling residues);

- enrichment of the uncoupled structural modal basis by the static response of the structure due to the unit forces on the non-linear degrees of freedom and enrichment of the uncoupled fluid basis by the static response of the fluid due to the presence of the structure and by taking into account the non-linear behaviour (Coupling + NL residues).

\subsection{Acoustic cavity}

The first example is an academic application to illustrate the non-linear coupling effects. Let us consider a thin plate $\left(0.654 \times 0.527 \times 0.003 \mathrm{~m}^{3}\right)$ with localized nonlinearities (17 at all) fixed on an acoustic cavity $\left(0.654 \times 0.527 \times 0.6 \mathrm{~m}^{3}\right)$ filled with air. Figure 2 shows the finite element model of the system. The model size is about 11000 dofs (3000 structural 8000 fluid dofs). The model frequency band is $[0-300 \mathrm{~Hz}]$; reduced model size is about 150 dofs against 11000 for the full model. Dissipation energy is introduced by modeling a proportional damping deduced from the first four structural mode with a damping ratio of $0.1 \%$. Structure is excited using an impact excitation of $500 \mathrm{~N}$ in a period of $10 \mathrm{~ms}$; Figure 3 shows the temporal and spectral representation of the impact.

The temporal moments for a period of $0.1 \mathrm{~s}$ of the structural displacement and the acoustic pressure for the considered model reduction strategies are presented in Tables 1 and 2. Structural displacement at non-linear dofs and prediction indicators are presented in Figures 4 and 5. The linear model response is also presented on the same figures to illustrate the impact of the non-linear effects.

In this application, results show the need to enrich the bases. More precisely, temporal moments for structural displacements show the need to take into account non-linear effects and temporal moments for acoustic pressure show the need to take into account the coupling effects. In both cases modal bases do not include enough information to properly represent the behaviour of the system.

In the case of strong coupling, when a heavy fluid as water is considered in the acoustic domain, the reduced order method should take into account heavy fluid be- 


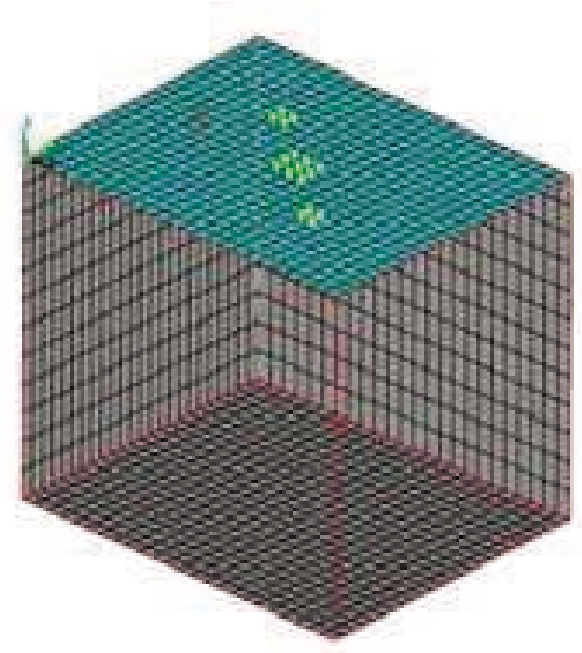

Figure 2. Acoustic cavity
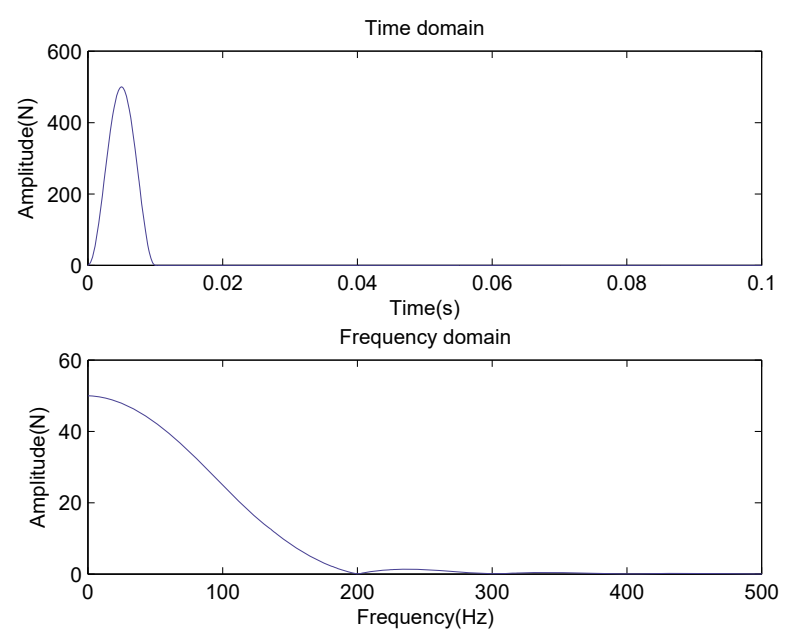

Figure 3. Impact spectrum

Table 1. Temporal moments for structural displacements

\begin{tabular}{|l|c|c|c|}
\hline & $T$ & $E$ & $D$ \\
\hline Full model & 0.0439 & $6.3637 \mathrm{e}-06$ & $8.48869 \mathrm{e}-04$ \\
\hline Modal basis & 0.0438 & $6.1156 \mathrm{e}-06$ & $8.4772 \mathrm{e}-04$ \\
Error / Full model (\%) & -0.2 & -3.9 & -0.1 \\
\hline Coupling residues & 0.0438 & $6.0208 \mathrm{e}-06$ & $8.4647 \mathrm{e}-04$ \\
Error / Full model (\%) & -0.2 & -5.4 & -0.3 \\
\hline NL residues & 0.0439 & $6.3331 \mathrm{e}-06$ & $8.4906 \mathrm{e}-04$ \\
Error / Full model (\%) & 0 & -0.5 & 0.02 \\
\hline Coupling + NL residues & 0.0439 & $6.3633 \mathrm{e}-06$ & $8.4882 \mathrm{e}-04$ \\
Error / Full model (\%) & 0 & -0.00 & -0.00 \\
\hline
\end{tabular}

Table 2. Temporal moments for acoustic pressure

\begin{tabular}{|l|c|c|c|}
\hline & $T$ & $E$ & $D$ \\
\hline Full model & 0.0529 & $2.7754 \mathrm{e}+06$ & $8.1539 \mathrm{e}-04$ \\
\hline Modal basis & 0.0538 & $2.8350 \mathrm{e}+06$ & $8.0777 \mathrm{e}-04$ \\
Error / Full model (\%) & 1.7 & 2.1 & -0.9 \\
\hline Coupling residues & 0.0532 & $2.7550 \mathrm{e}+06$ & $8.0692 \mathrm{e}-04$ \\
Error / Full model (\%) & 0.6 & -0.7 & -1.03 \\
\hline NL residues & 0.0540 & $2.8928 \mathrm{e}+06$ & $8.1152 \mathrm{e}-04$ \\
Error / Full model (\%) & 2.1 & 4.2 & -0.5 \\
\hline Coupling + NL residues & 0.0529 & $2.7745 \mathrm{e}+06$ & $8.1512 \mathrm{e}-04$ \\
Error / Full model (\%) & 0 & -0.03 & -0.03 \\
\hline
\end{tabular}




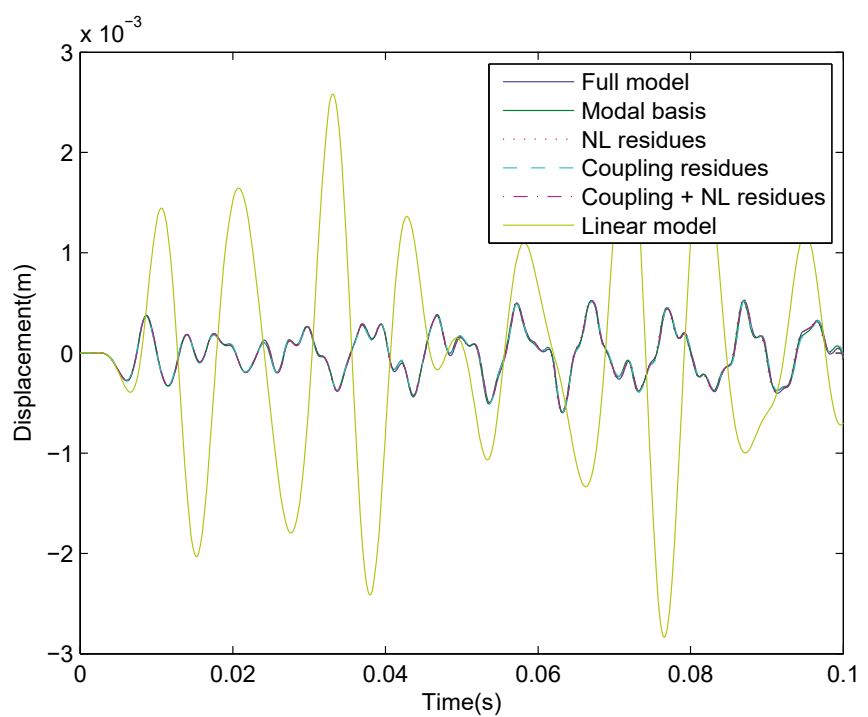

a)

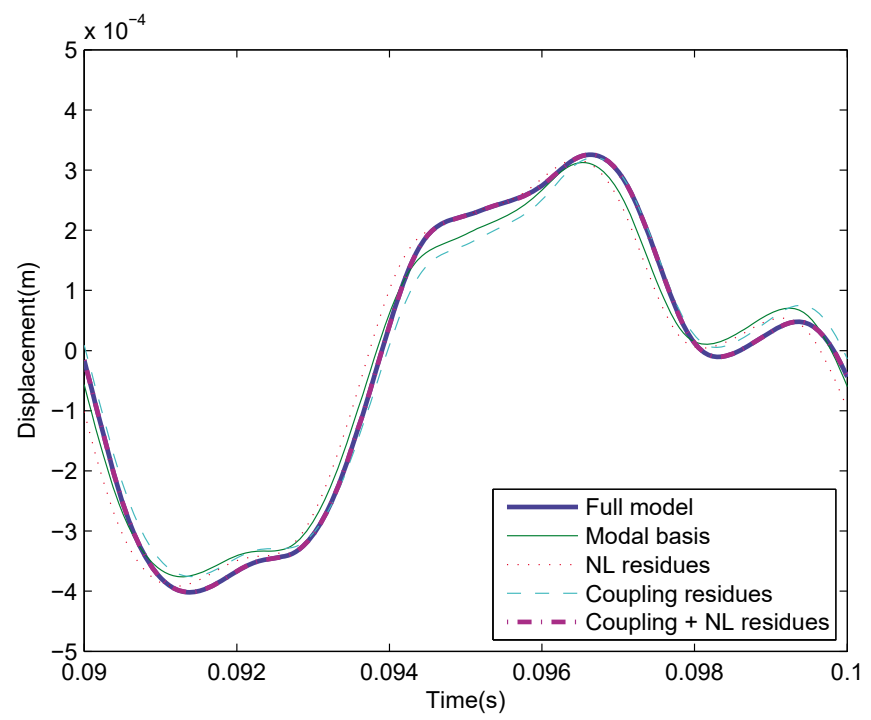

b)

Figure 4. Displacement as a function of time. a) in a period of $0.1 s$ with the linear model, b) in the period [0.09-0.1]s

haviour as it was mentioned before. Figure 6 presents the predictive indicators of the full model compared to the reduced model with and without taking into account the heavy fluid. Results show the need to take into account heavy fluid effects. The nonconvergence of the model that do not consider the heavy fluid effect compared to the full model can be observed in Figure 6. 

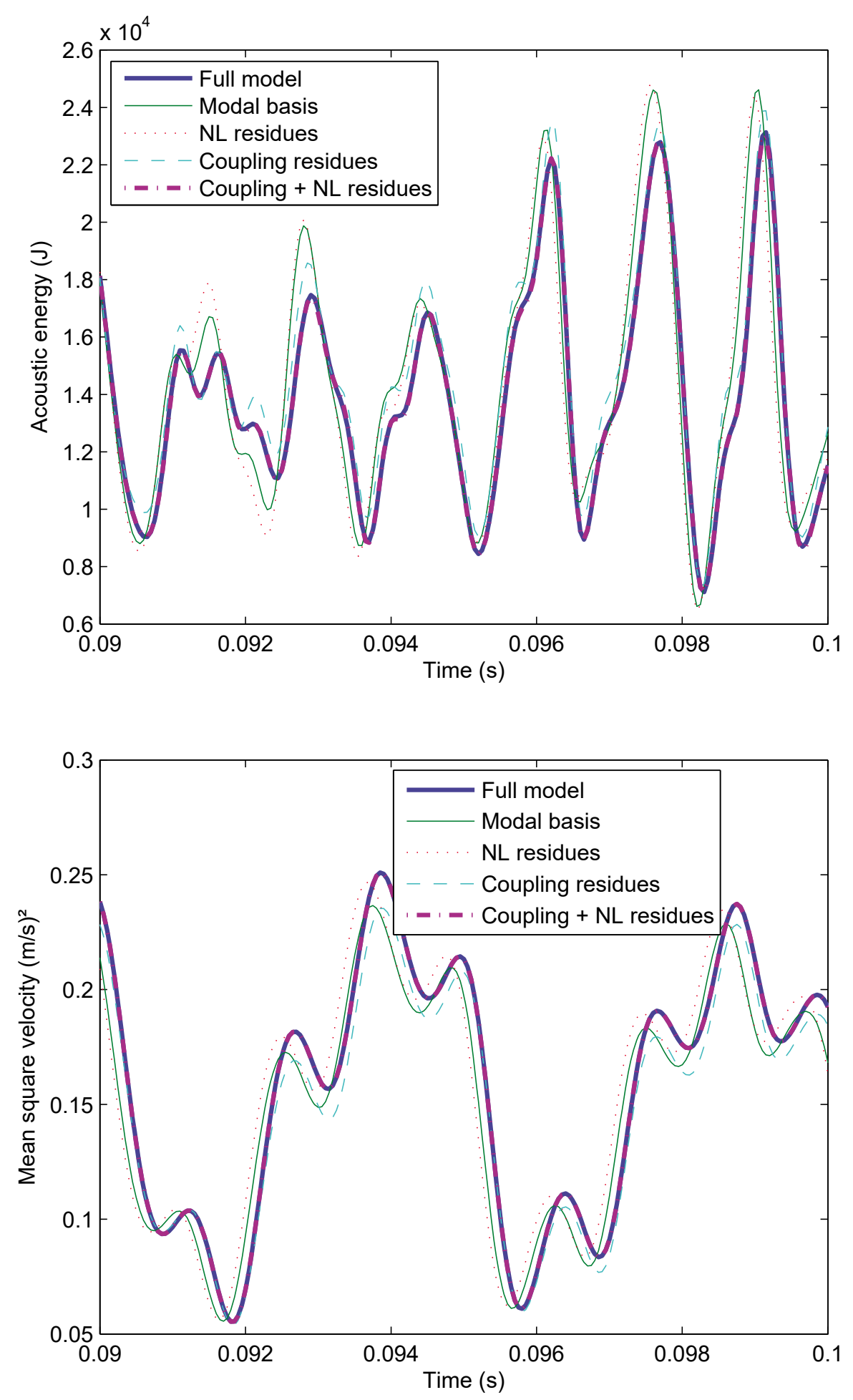

Figure 5. Prediction indicators - light coupling 

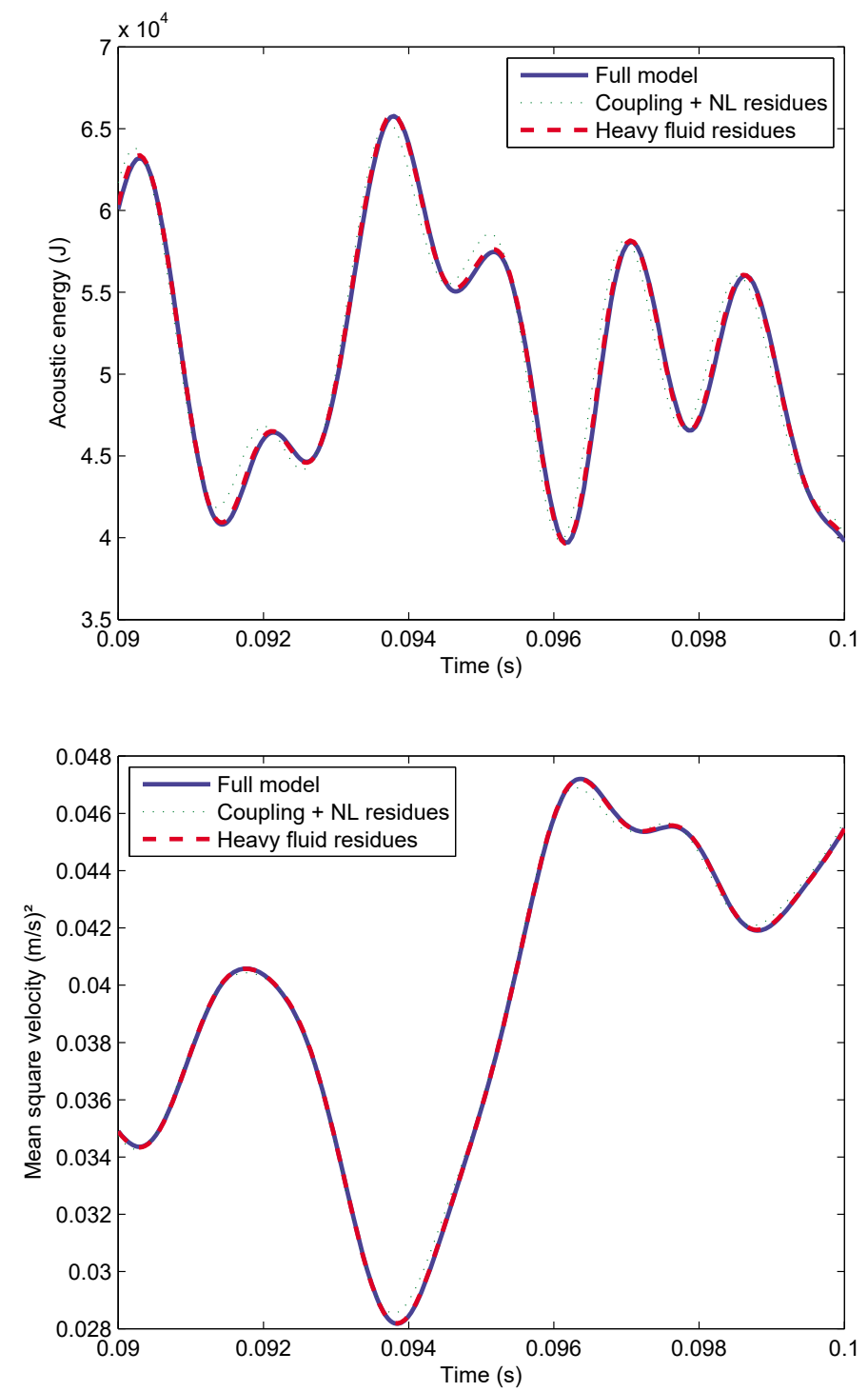

Figure 6. Prediction indicators - heavy coupling

\subsection{Exhaust}

Let us consider an air cavity in a fold exhaust suspended with 18 non-linear springs. The exhaust dimensions are $L 1+L 2+L 3=0.2+0.25+0.2 m$, $R 1=R 3=0.05 \mathrm{~m}$ and $R 2=0.125 \mathrm{~m}$ (Figure 7). The finite element model contains 8500 dofs ( 5000 for the structure and 3500 for the fluid). The model is valid in the frequency band $[0-450 \mathrm{~Hz}]$. A sweep sine in the frequency band of interest is used to excite the structure with a $1 N$ amplitude. Figure 8 shows the temporal and spectral representations of the excitation. Proportional damping is used to introduce dissipation in the model. It is deduced from the first four structural damped modes with a damping ratio of $0.1 \%$. Reduced order methods presented previously are compared to the full model. The reduced model size is 200 dofs. The temporal moments of structural displacements and acoustic pressure are presented in Tables 3 and 4 . The acoustic energy and the mean square velocity are presented in Figures 9 and 10. 


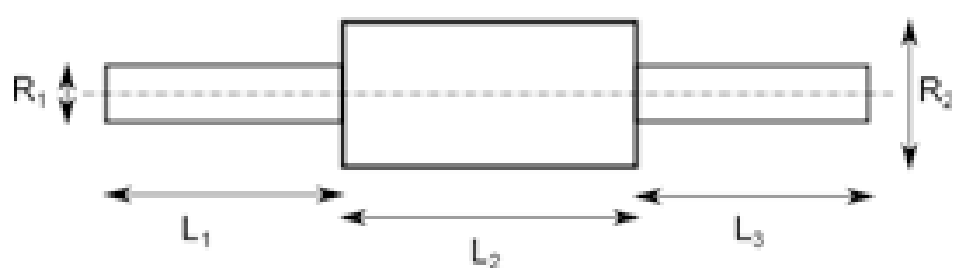

Figure 7. Exhaust
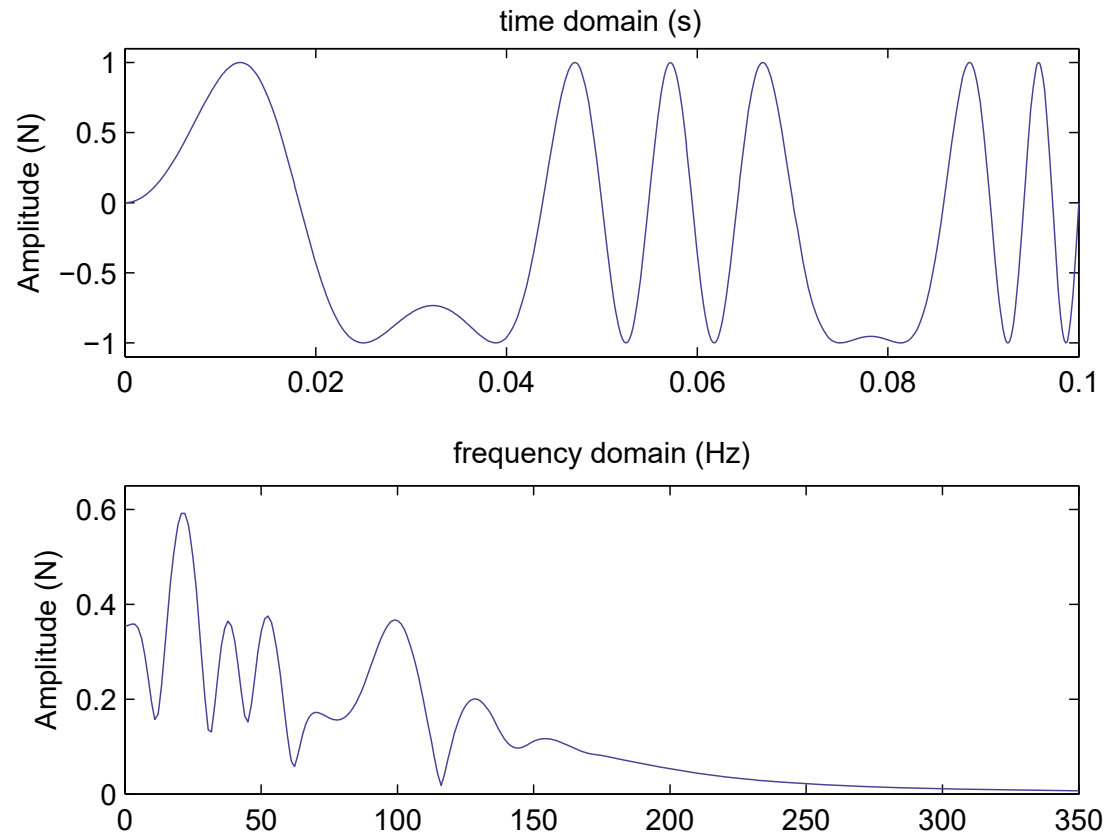

Figure 8. Excitation sine sweep

Table 3. Temporal moments for structural displacements

\begin{tabular}{|l|c|c|c|}
\hline & $D$ & $E$ & $T$ \\
\hline Full model & $4.1211 \mathrm{E}-04$ & $1.1772 \mathrm{E}-04$ & 0.0650 \\
\hline Modal basis & $4.1025 \mathrm{E}-04$ & $1.1633 \mathrm{E}-04$ & 0.0646 \\
Error / Full model (\%) & -0.5 & -1.2 & -0.6 \\
\hline Coupling residues & $4.1026 \mathrm{E}-04$ & $1.2074 \mathrm{E}-04$ & 0.0651 \\
Error / Full model (\%) & -0.4 & 2.6 & 0.2 \\
\hline NL residues & $4.1018 \mathrm{E}-04$ & $1.1627 \mathrm{E}-04$ & 0.0646 \\
Error / Full model (\%) & -0.5 & -1.2 & -0.6 \\
\hline Coupling + NL residues & $4.1197 \mathrm{E}-04$ & $1.1739 \mathrm{E}-04$ & 0.0649 \\
Error / Full model (\%) & -0.03 & -0.3 & -0.2 \\
\hline
\end{tabular}


Table 4. Temporal moments for acoustic pressure

\begin{tabular}{|l|c|c|c|}
\hline & $D$ & $E$ & $T$ \\
\hline Full model & $4.3529 \mathrm{E}-04$ & $1.2554 \mathrm{E}+05$ & 0.0670 \\
\hline Modal basis & $4.2964 \mathrm{E}-04$ & $1.2277 \mathrm{E}+05$ & 0.0663 \\
Error / Full model (\%) & -1.3 & -2.2 & -1.0 \\
\hline Coupling residues & $4.2312 \mathrm{E}-04$ & $1.4276 \mathrm{E}+05$ & 0.0676 \\
Error / Full model (\%) & -2.8 & 13.8 & 0.9 \\
\hline NL residues & $4.2961 \mathrm{E}-04$ & $1.2276 \mathrm{E}+05$ & 0.0663 \\
Error / Full model (\%) & -1.3 & -2.2 & -1.0 \\
\hline Coupling + NL residues & $4.3179 \mathrm{E}-04$ & $1.2583 \mathrm{E}+05$ & 0.0669 \\
Error / Full model (\%) & -0.8 & 0.2 & -0.1 \\
\hline
\end{tabular}

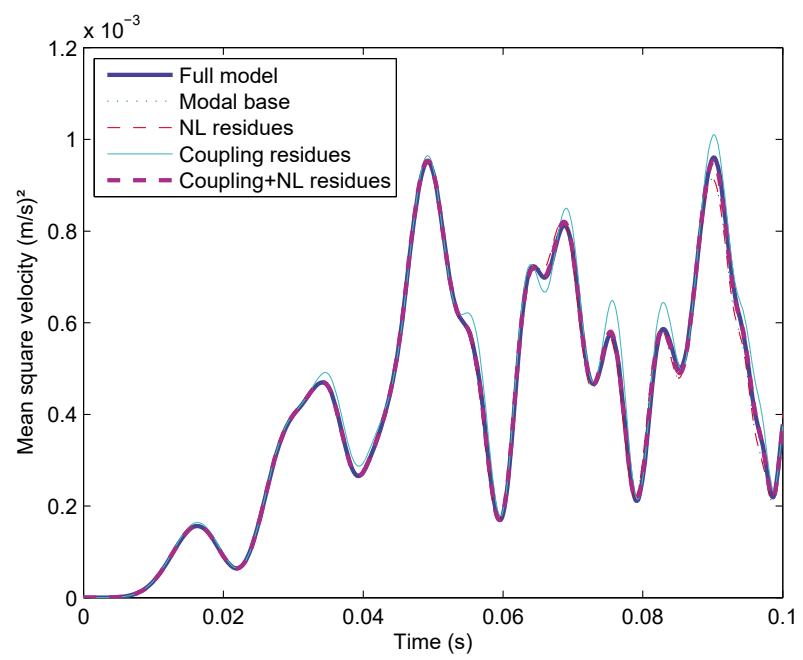

a)

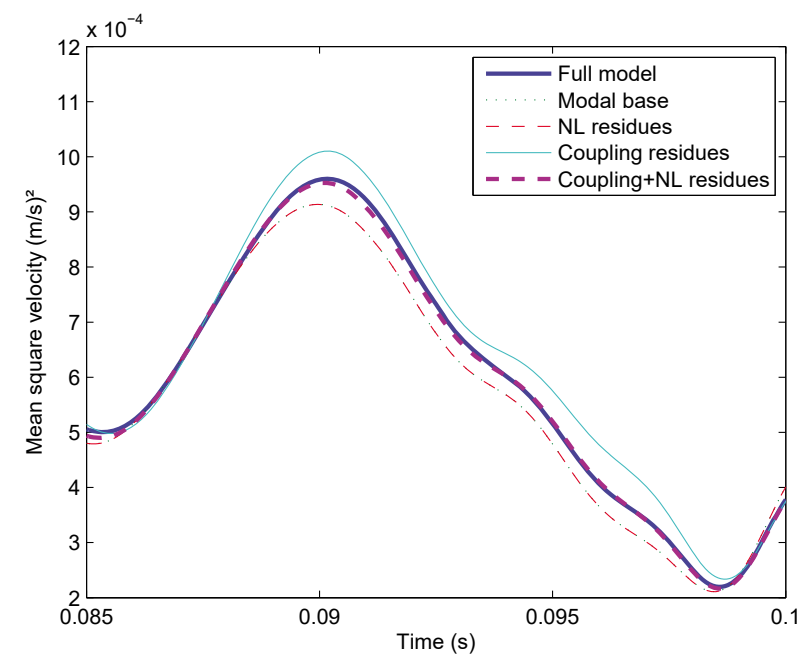

b)

Figure 9. Mean square velocity - a) $t=[0-0.1] s, b) t=[0.085-0.1] s$ 


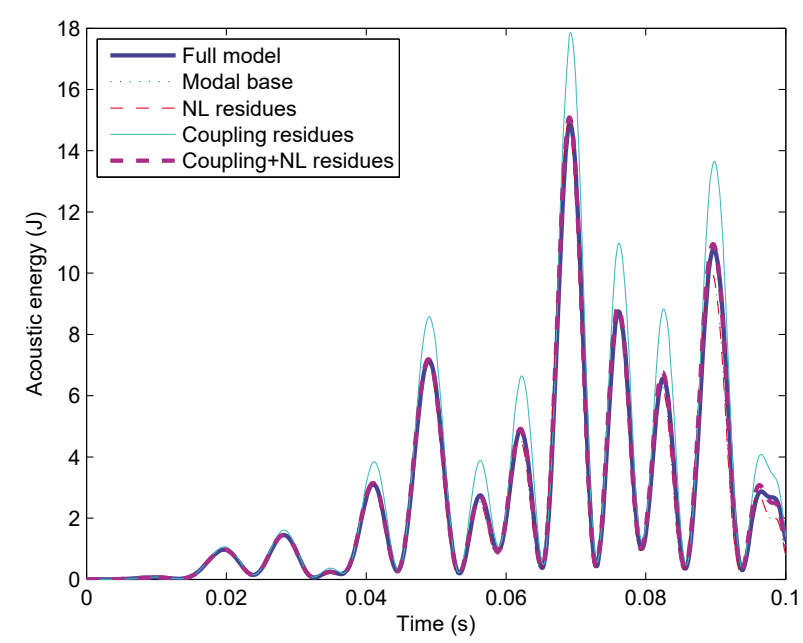

a)

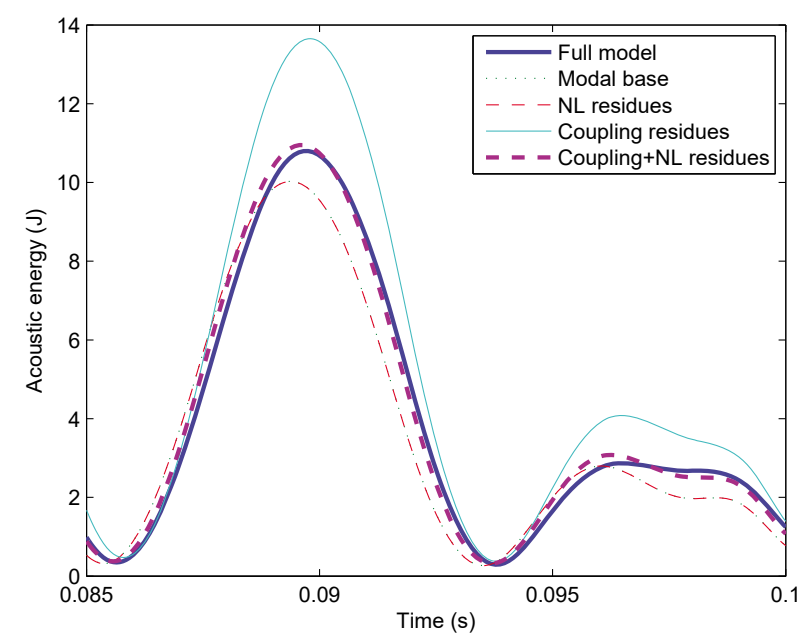

b)

Figure 10. Acoustic energy - a) $t=[0-0.1] s, b) t=[0.085-0.1] s$

This application illustrates once again the poor efficiency of the uncoupled projecting bases. Residual terms are required to ensure good convergence, but enrichment can also sometimes lead to divergence of the solution. This is the case in the simulation with the reduced model using the coupling effects. In the same time, enriching using only non-linear effects seems to be insufficient. It predicts the same behaviour as the reduced model using the modal basis.

Both applications show the need for a reduced model tool independently of the excitation type. In both cases the necessity of enriching to ensure good converging propreties has been illustrated. Using the reduced model tool provides a benefit in time consuming: in these applications the calculating time was divided by 10 compared to the full model. Concerning the result precision, the temporal moments show the necessity on enriching the bases. In fact, according to the response, enriching should 
take into account non-linear and coupling effects to converge to the solution of the full model. In the case of heavy fluid, numerical results show the limit of the proposed method and the need to consider the heavy fluid in the reduced basis to ensure good predicting.

\section{Conclusion}

A reduced order method adapted to non-linear coupling problems has been presented. It is required for localized geometrical non-linear problems and is in the process of being extended to other types of non-linearities. It consists in enriching the uncoupled modal basis by residues taking into account non-linearity and coupling conditions. It was mentioned that associating conditions is very important to ensure convergence. Examples in the temporal space with different types of excitations have been studied. An application of the approach in the frequency domain is conceivable using the harmonic balance method adapted for geometric non-linearities.

\section{References}

Amabili M., Nonlinear Vibrations And Stability Of Shells And Plates, Cambridge, 2008.

Amabili M., Touzé C., " Reduced-order models for nonlinear vibrations of fluid-filled circular cylindrical shells: Comparison of POD and asymptotic nonlinear normal modes methods", Journal of Fluids and Structures, vol. 23, p. 885-903, 2007.

Bathe K., Finite Element Procedures in Engineering Analysis, Prentice Hall, 1982.

Géradin M., Rixen D., Mechanical Vibrations: Theory and Applications to Structural Dynamics, John Wiley \& Sons Ltd, 1997.

Irons B., " Role of part-inversion in fluid-structure problems with mixed variables", AIAA Journal, vol. 8, p. 568, 1970.

Masson G., Ait-Brik B., Bouhaddi N., Cogan S., " Component mode synthesis (CMS) based on an enriched Ritz approach for efficient structural optimization", Journal of Sound and Vibration, vol. 296, p. 845-860, 2006.

Morand H., Ohayon R., Interactions Fluides-Structures, MASSON, 1992.

Nayfeh A. H., Mook D. T., Nonlinear Oscillations, Wiley Classics Library, 1995.

Pérignon F., Vibrations Forcées De Structures Minces, 'Elastiques, Non-Linéaires, PhD thesis, Université de la Méditerranée (Aix-Marseille II), 2004.

Soares-Jr D., von Estorff O., Mansur W., " Efficient non-linear solid-fluid interaction analysis by an iterative BEM/FEM coupling", International Journal for Numerical Methods in Engineering, vol. 64, p. 1416-1431, 2005.

Tran Q., Analyse robuste et Optimisation de problème vibroacoustiques avec interfaces absorbantes, PhD thesis, Université de Franche-Comté, 2009.

Tran Q. H., Ouisse M., Bouhaddi N., “ A robust component mode synthesis method for stochastic damped vibroacoustics", Mechanical Systems and Signal Processing, vol. 24, p. 164$181,2010$. 